\title{
Fingerprint Verification with Non-linear Composite Correlation Filters
}

\author{
Saúl Martínez-Díaz and Javier A. Carmona-Troyo \\ Instituto Tecnológico de La Paz, División de Estudios de Posgrado e Investigación, \\ Boulevard Forjadores de Baja California Sur No.4720, La Paz BCS, México \\ smdiaz@marinos.itlp.edu.mx, jcarmona@marinos.itlp.edu.mx
}

\begin{abstract}
Fingerprint recognition has been used from many years for identification of persons. However, conventional fingerprint recognition systems might fail with poor quality, noisy or rotated images. Recently, novel non-linear composite filters for correlation-based pattern recognition have been introduced. The filters are designed with information from distorted versions of reference object to achieve distortion-invariant recognition. Besides, a non-linear correlation operation is applied among the filter and the test image. These kinds of filters are robust to non-Gaussian noise. In this paper we apply non-linear composite filters for fingerprint verification. Computer simulations show performance of proposed filters with distorted fingerprints. In addition, in order to illustrate robustness to noise, filters were tested with noisy images.
\end{abstract}

Keywords: Fingerprint verification, nonlinear filters, correlation filters, pattern recognition.

\section{Introduction}

One of the most important biometric person identification techniques is based on fingerprint recognition. Fingerprints are unique and unchangeable to each individual; for this reason had been used since many years ago for this purpose. Basically two tasks are performed with fingerprint recognition systems: identification of a person or verification of his/her identity. In the first case the system searches into a database, if a similar fingerprint is found the person is identified. In the second case, the system verifies if a person is who he/she claims to be.

Generally, fingerprint recognition systems are based on minutiae (ridge endings and bifurcations) extraction [1]. With these methods preprocessing is required to remove noise, enhance the image and extract the features of interest. Extracted features are employed to identify the fingerprint.

Other methods that use local and global features of fingerprints have been proposed [2], [3], [4]. However, those techniques do not use all available information from images. Besides, with the above mentioned techniques, orientation of images is crucial in the process. Therefore rotation, poor quality and noisy images make difficult the recognition. 
Another way to carry out fingerprints recognition is by correlating the entire test image with a single template or filter [5]. When the test image is equal to the template, correlation output is high (typically one); in other case correlation is low. Figures 1(a) and 1(b) show examples of correlation planes for two equal images and for two different images, respectively. Note that when images perfectly match a sharp peak is observed at origin of coordinates. A threshold can be established at the output to determinate whether the tested fingerprint is authentic or not. Correlation methods are shift-invariant and exploit all information from images. Moreover, by using several samples of the expected distortions of objects is possible to design distortioninvariant filters. The training images are synthesized in a single template and the test image is correlated with such template.
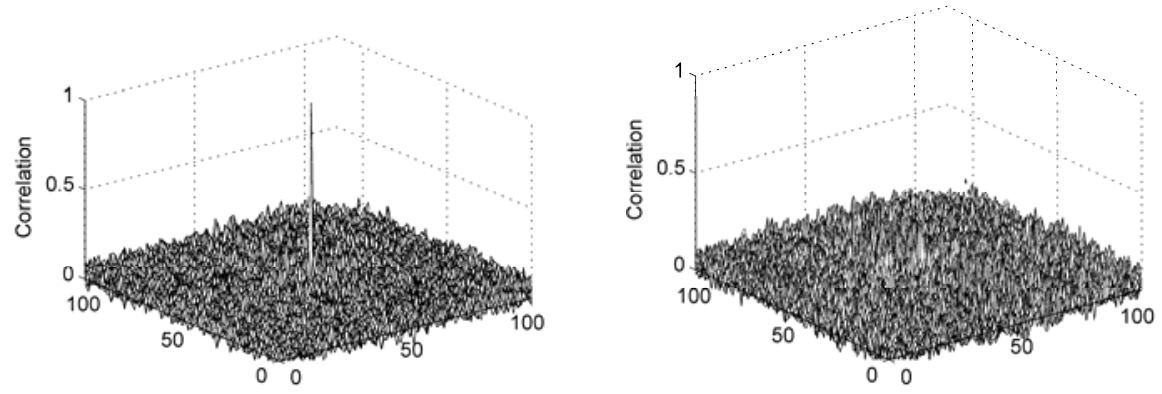

Fig. 1. Correlation planes obtained by a) exact match among images and b) not match among images

Normally, correlation filters are designed and optimized in a linear way. Linear filters used to be robust to Gaussian noise; however, real images are often corrupted by non-Gaussian noise. In practice, non-linear filters are more robust even for slight deviations from the Gaussian distribution. This kind of filters provides solutions in many cases where linear filters are inappropriate. In this paper we propose the use of novel non-linear composite filters. The filters are based on morphological and rankorder operations; its robustness to non-Gaussian noise has been shown.

The paper is organized as follows: In section 2 we review traditional composite filters. In section 3 we introduce non-linear filtering and proposed filters. In section 4 computer simulations are provided and discussed. Section 5 summarizes our conclusions.

\section{Composite Filters}

The simplest correlation filter, called Matched filter (MF), is a single image used as template, which is correlated with the test image [6]. It is known that the MF is very sensitive to small distortions of the object caused by variations in scale, rotation, or point of view. In order to overcome these problems, composite filters based on synthetic discriminant functions (SDF) were introduced [7]. 


\subsection{Synthetic Discriminant Function Filters}

Conventional SDF filters are a linear combination of MFs for different patterns. The coefficients of the linear combination are chosen to satisfy a set of constraints on the filter output requiring a previously specified value for each pattern used.

Suppose there are $N$ training images from a true class, each image contains $d$ pixels. The $2 \mathrm{D}$ arrays of the images are converted into $1 \mathrm{D}$ column vector by lexicographical ordering. These vectors are the columns of a matrix $\mathrm{R}$ of size $d \times N$. The column vector u contains $N$ elements, which are the desired values of the output correlation peaks corresponding to each training image. If the matrix $\left(R^{+} R\right)$ is nonsingular, the conventional SDF filter can be expressed as follows:

$$
\mathrm{h}_{S D F}=\mathrm{R}\left(\mathrm{R}^{+} \mathrm{R}\right)^{-1} \mathrm{u},
$$

where superscript + means conjugate transpose. The main shortcoming of the linear SDF filters is appearance of sidelobes due to the lack of control over the whole correlation plane. In order to reject known objects from a false class, these objects can be included in the training set by setting zero in the corresponding values of vector $\mathrm{u}$.

\subsection{Minimum Average of Correlation Energy Filter}

With the intention of suppress false correlation peaks, minimum average of correlation energy (MACE) filters were proposed [8]. MACE filters minimize the average correlation energy of the correlation outputs for a set of training images, satisfying at the same time the correlation peak constraints at the origin. Suppose that there are $N$ training images, each image with $d$ pixels. First, the 2D Fourier transform is performed on each training image and converted into $1 \mathrm{D}$ column vector. Then, a matrix $\mathrm{X}$ with $N$ columns and $d$ rows is constructed. The columns of $\mathrm{X}$ are given by the vector version of each transformed image. The frequency response of the MACE filter can be expressed as

$$
\mathrm{h}_{\text {MACE }}=\mathrm{D}^{-1} \mathrm{X}\left(\mathrm{X}^{+} \mathrm{D}^{-1} \mathrm{X}\right)^{-1} \mathrm{u},
$$

where the column vector $\mathrm{u}$ contains desired correlation peak values of the training images and the $d \mathrm{x} d$ diagonal matrix $\mathrm{D}$ contains the average power spectrum of the training images.

\subsection{Optimal Tradeoff Filters}

MACE filters maximize peak sharpness by minimizing correlation energy. However, tolerance to noise is not considered. In order to include noise tolerance, optimal tradeoff synthetic discriminant function (OTSDF) filters were introduced [9]. OTSDF filters allow a tradeoff among peak sharpness and noise tolerance by minimizing at the same time correlation energy and the output variance of correlation peak when the input images of the training set are corrupted by noise. The frequency response of the OTSDF filter can be expressed as

$$
\mathrm{h}_{\text {OTSDF }}=\mathrm{P}^{-1} \mathrm{X}\left(\mathrm{X}^{+} \mathrm{P}^{-1} \mathrm{X}\right)^{-1} \mathrm{u},
$$


where $\mathrm{P}=\mu \mathrm{D}+(1-\mu) \mathrm{C}, \mu \in[0,1]$. The $d \mathrm{x} d$ diagonal matrix $\mathrm{D}$ contains the average power spectrum of the training images. $\mathrm{C}$ is a matrix of covariance obtained from noise realizations. The column vector $\mathrm{u}$ contains desired correlation peak values of the training images.

\section{Nonlinear Filtering}

Traditionally correlation-based filters use a linear correlation operation derived from minimization of the mean squared error (MSE). The correlation is computed between an input image and a shifted version of the target. On the other hand, minimization of the mean absolute error (MAE) leads to a nonlinear operation, called morphological correlation, which is computed as a sum of minima. This criterion is more robust when the noise has even slight deviations from the Gaussian distribution, and produces a sharper peak at the origin [10]. Also, local adaptive correlations based on rank order operations were proposed to improve recognition in images with non-Gaussian noise [11]. Recently, novel non-linear synthetic discriminant function filters (N-SDF) were proposed [12]. The filters are designed by applying logical operations among a set of training objects. Various objects to be recognized and rejected can be incorporated in the template synthesis. The morphological correlation is computed between the template and a test scene. The correlation is locally normalized to yield a desired output value. It was shown that nonlinear filters yield maximum correlation with objects utilized in the template synthesis. Besides, filters are robust in images corrupted by non- Gaussian noise.

\subsection{Morphological Correlation}

The proposed filtering is a locally adaptive processing of the signal in a moving window. The moving window is a spatial neighborhood containing pixels surrounding the central window pixel geometrically. The neighborhood is referred to as the $W$ neighborhood. The shape of the $W$-neighborhood is similar to the region of support of the target. The size of the neighborhood is referred to as $|W|$, and it is approximately taken as the size of the target.

Let $\{T(k, l)\}$ and $\{S(k, l)\}$ be a target image and a test scene respectively, both with $Q$ levels of quantization. Here $(k, l)$ are the pixel coordinates. The local nonlinear correlation derived from the MAE criterion between a normalized input scene and a shifted version of the target at coordinates $(k, l)$ can be defined as

$$
C(k, l)=\sum_{m, n \in W} \operatorname{MIN}[a(k, l) S(m+k, n+l)+b(k, l), T(m, n)]
$$

where the sum is taken over the $W$-neighborhood. $a(k, l)$ and $b(k, l)$ are local normalizing coefficients, which take into account unknown illumination and bias of the target, respectively. The optimal coefficients with respect to the MAE can be estimated by minimizing the MSE between the window signal and the target. Their explicit estimates are given by: 


$$
\begin{gathered}
a(k, l)=\frac{\sum_{m, n \in W} T(m, n) \cdot S(m+k, n+l)-|W| \cdot \bar{T} \cdot \bar{S}(k, l)}{\sum_{m, n \in W}(S(m+k, n+l))^{2}-|W| \cdot(\bar{S}(k, l))^{2}}, \\
b(k, l)=\bar{T}-a(k, l) \cdot \bar{S}(k, l),
\end{gathered}
$$

here $\bar{T}$ and $\bar{S}(k, l)$ are the average of the target and local window signal over the $W$-neighborhood at the $(k, l)$ 'th window position, respectively.

\subsection{Nonlinear Synthetic Discriminant Function Filters}

According to the threshold decomposition concept [13], a gray-scale image $X(k, l)$ can be represented as a sum of binary slices:

$$
X(k, l)=\sum_{q=1}^{Q-1} X^{q}(k, l)
$$

where $\left\{X^{q}(k, l), q=1, \ldots Q-1\right\}$ are binary slices obtained by decomposition of the image with a threshold $q$ as follows:

$$
X^{q}(k, l)=\left\{\begin{array}{l}
1, \text { if } \quad X(k, l) \geq q \\
0, \quad \text { otherwise }
\end{array} .\right.
$$

Now, assume that there are $N$ objects from the true class $\left\{T_{i}(k, l), i=1 \ldots N\right\}$ and $M$ objects from the false class $\left\{P_{j}(k, l), j=1 \ldots M\right\}$. First, binary images are obtained by threshold decomposition of the training set. Next we construct the non-linear synthetic discriminant function filter (N-SDF) as logical combinations of the binary images. The composite filter can be expressed as:

$$
\left.H_{N S D F}(k, l)=\sum_{q=1}^{Q-1}\left[\bigcap_{i=1}^{N} T_{i}^{q}(k, l)\right] \bigcap \overline{\bigcup_{j=1}^{M} P_{j}^{q}(k, l)}\right], \quad i=1 \ldots N, j=1 \ldots M
$$

where $\left\{T_{i}^{q}(k, l), q=1, \ldots Q-1, i=1, \ldots N\right\}$ and $\left\{P_{j}^{q}(k, l), q=1, \ldots Q-1, j=1, \ldots M\right\}$ are binary slices obtained by threshold decomposition from corresponding training images of true and false classes respectively. $U$ and $\bigcap$ represent the logical union and intersection, respectively. The neighborhood $W$ is taken as the region of support of the composite filter. Finally, the nonlinear correlation in equation (4) is computed among the test image and the composite filter. The result is normalized by $u / s$. Here $u$ is the desired value at the correlation output, and

$$
s=\sum_{k, l \in W} H_{N S D F}(k, l)
$$


It can be shown that the composite correlation yields the value $u$ at output correlation for objects belonging to the true class, while the output correlation peak for the false class objects is zero. Known false class images are normalized respect the target by using equations (5) and (6), before synthesize the template.

\section{Computer Simulations}

In this section computer simulation results obtained with the proposed filters are presented. A set of 200 fingerprints was utilized in the experiments. All images are $115 \times 115$ pixels at 256 levels of quantization. The performance of nonlinear filters is compared with that of MACE and OTSDF filters. For the OTSDF filter white noise is assumed and $\mu$ is set to 0.9 . Figure 2(a) shows the fingerprint to be recognized (target) and figure 2(b) shows an impostor's fingerprint. Then N-SDF, OTSDF and MACE filters were designed with five rotated versions of target $(-4,-2,02$ and 4 degrees) and five known false fingerprints.

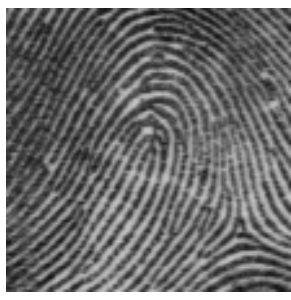

(a)

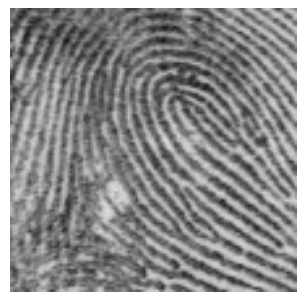

(b)

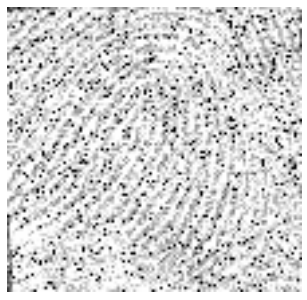

(c)

Fig. 2. (a) Fingerprint to be recognized (target). (b) False fingerprint. (c) An example of target corrupted by mixed additive Gaussian and impulsive noise. The mean and standard deviation of additive noise are 120 and 30 respectively. The probability of impulsive noise is 0.1 .

As previously mentioned, correlation between filters and the versions of target used to synthesize the template are equal to one. Now, correlation was executed between filters and 194 unknown false fingerprints. Then, the minimum value at which all false objects are rejected with all filters was selected as threshold. Such threshold was set to 0.8 .

Next, in order to test robustness to noise, correlation among composite filters and rotated versions of noisy target was computed. The noise was a mix of additive Gaussian and impulsive (salt and pepper) noise. The mean and standard deviation of additive noise were 120 and 40, respectively. The probability of impulsive noise was 0.1 with equal probability of occurrence for negative and positive impulses. Figure 2(c) shows an instance of target corrupted by mixed. To guarantee statistically correct results, 30 statistical trials of each experiment for different realizations of random processes were performed. Figure 3 shows performance of the filters. As can be seen, $\mathrm{N}-\mathrm{SDF}$ filter is able to detect the target in all cases, even when scene is corrupted with non-Gaussian noise. 


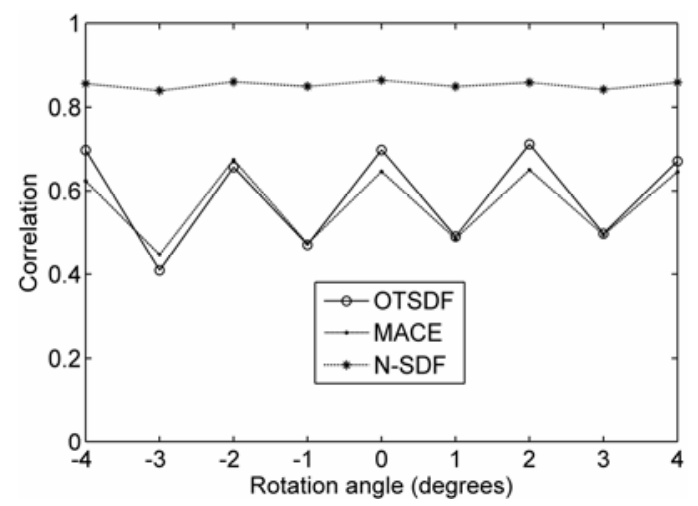

Fig. 3. Correlation output of MACE, OTSDF and N-SDF filters for rotated versions of target corrupted with mixed additive and impulsive noise

Table 1. Performance of composite filters in terms of maximum correlation peak when target is incomplete

\begin{tabular}{cccr}
\hline$\%$ Of original image & MACE & $\begin{array}{r}\text { Correlation peak } \\
\text { OTSDF }\end{array}$ & N-SDF \\
\hline $96 \%$ & 1.000 & 1.000 & 1.000 \\
$93 \%$ & 0.781 & 0.801 & 0.997 \\
$90 \%$ & 0.681 & 0.690 & 0.992 \\
$86 \%$ & 0.656 & 0.662 & 0.975 \\
$83 \%$ & 0.648 & 0.653 & 0.945 \\
$80 \%$ & 0.633 & 0.640 & 0.911 \\
$77 \%$ & 0.617 & 0.628 & 0.880 \\
$74 \%$ & 0.562 & 0.567 & 0.847 \\
$71 \%$ & 0.550 & 0.554 & 0.795 \\
$68 \%$ & 0.540 & 0.542 & 0.762 \\
\hline
\end{tabular}

Often, because of fingerprint pressure differences, images are incomplete. For this reason we test performance of filters with incomplete fingerprints. First, pixels are removed at each edge of target. Then, correlation between designed filters and incomplete target is computed. Results are presented in table 1 . Column 1 is the remaining percentage of original image. Column 2, 3 and 4 are the maximum correlation peaks for MACE, OTSDF and N-SDF filters, respectively. Note that correlation with N-SDF filter decreases slower than correlation with MACE and OTSDF filters. $\mathrm{N}-\mathrm{SDF}$ yields a correlation value above threshold with less than 75 percent of original image.

\section{Conclusions}

In this paper, composite nonlinear filters for fingerprint verification were proposed. The filters are designed as a logical combination of given training images. Various properties of filters were tested. Their recognition performance and noise robustness 
were compared with those of conventional linear composite filters. Computer simulations illustrated an improvement in recognition of distorted fingerprints in heavy nonGaussian noise situations, when the proposed filters were used. As well, proposed filters were capable of recognize even incomplete target. Further simulations can be done in order to test extensively non-linear filters.

\section{References}

1. Maltoni, D., Maio, D., Jain, A.K., Prabhakar, S.: Handbook of Fingerprint Recognition. Springer, London (2009)

2. Jain, A.K., Prabhakar, S., Hong, L., Pankanti, S.: Filterbank-Based Fingerprint Matching. IEEE T. on Image Process. 9, 846-859 (2000)

3. Ross, A., Reisman, J., Jain, A.: Fingerprint Matching Using Feature Space Correlation. In: Tistarelli, M., Bigun, J., Jain, A.K. (eds.) ECCV 2002. LNCS, vol. 2359, pp. 48-57. Springer, Heidelberg (2002)

4. Cappelli, R., Maio, D., Maltoni, D., Nanni, L.: A Two-Stage Fingerprint Classification System. In: Workshop on Biometrics Methods and Applications, pp. 95-99. ACM, California (2003)

5. Venkataramani, K., Vijaya-Kumar, B.V.K.: Fingerprint Verification Using Correlation Filters. In: Kittler, J., Nixon, M.S. (eds.) AVBPA 2003. LNCS, vol. 2688, pp. 886-894. Springer, Heidelberg (2003)

6. VanderLugt, A.B.: Signal Detection by Complex Filtering. IEEE Trans. Inf. Theory. 10, 135-139 (1964)

7. Hester, C.F., Casasent, D.: Multivariant Technique for Multiclass Pattern Recognition. Appl. Opt. 19, 1758-1761 (1980)

8. Mahalanobis, A., Vijaya-Kumar, B.V.K., Casasent, D.: Minimum Average Correlation Energy Filters. Appl. Opt. 31, 1823-1833 (1987)

9. Refregier, P.: Filter Design for Optical Pattern Recognition: Multicriteria Optimization Approach. Optical Society of America 15, 854-856 (1990)

10. Maragos, P.: Morphological Correlation and Mean Absolute Error Criteria. In: Proc. Conf. IEEE Trans. Acoust. Speech Signal Process., pp. 1568-1571 (1989)

11. Kober, V., Alvarez-Borrego, J., Ovseyevich, I.A.: Adaptive Rank Order Correlations. Pattern Recognition and Image Analysis 14, 33-39 (2004)

12. Martínez-Díaz, S., Kober, V.: Nonlinear Synthetic Discriminant Function Filters for Illumination-Invariant Pattern Recognition. Opt. Eng. 47, 067201 (2008)

13. Fitch, J.P., Coyle, E.J., Gallagher Jr., N.C.: Median Filtering by Threshold Decomposition. IEEE Trans. Acoust. Speech Signal Process., 1183-1188 (1984) 